\title{
Acrylamide-induced adverse cerebellar changes in rats: possible oligodendrogenic effect of omega 3 and green tea
}

\author{
R.A. Imam, H.N. Gadallah \\ Department of Anatomy and Embryology, Faculty of Medicine, Cairo University, Cairo, Egypt \\ [Received: 13 September 2018; Accepted: 10 December 2018]
}

Background: Humans are widely exposed to acrylamide (ACR) and its neurotoxicity is a significant public health issue attracting wide attention. The aim of the study was to investigate ACR-induced adverse cerebellar changes in rats and study the possible oligodendrogenic effect of omega 3 and green tea.

Materials and methods: Twenty-four adult albino rats weighing 150-200 g were randomly divided into four equal groups (6 rats each): control group (Group I), the rats that received $A C R 45 \mathrm{mg} / \mathrm{kg} /$ day (Group II), the rats that received $A C R$ concomitant with omega 3 at a dosage of $200 \mathrm{mg} / \mathrm{kg} /$ day (Group III), the rats that received $A C R$ concomitant with green tea dissolved in drinking water at a dosage of $5 \mathrm{~g} / \mathrm{L}$ (Group IV). The rats were euthanized after 8 weeks of the experiment. Malondialdehyde (MDA) and glutathione (GSH) were measured in cerebellar homogenates. Sections of $5 \mu \mathrm{m}$ thickness from specimens from the cerebellum were stained with haematoxylin and eosin, silver stain and immunohistochemical stains: platelet-derived growth factor alpha (PDGFa; for oligodendrocytes), glial fibrillary acidic protein (GFAP; for astrocytes) and BCL2 (antiapoptotic).

Results: Omega 3 and green tea had improved MDA and GSH as compared to the ACR group. Histologically, the ACR group showed variable degrees of cellular degeneration. Omega 3 had induced oligodendrogenesis in Group III. The optical density of silver stain was significantly $(p<0.05)$ increased in Groups III and IV as compared to the ACR group. Area per cent of positive PDGF $\alpha$ was significantly increased in the ACR + omega 3 group as compared to the ACR group. Area per cent of positive GFAP was significantly decreased in Groups III and IV as compared to the ACR group. Area per cent of positive BCL2 was significantly increased in the omega 3-trated group as compared to the ACR group.

Conclusions: Concomitant administration of omega 3 or green tea with $A C R$ might mitigate the adverse cerebellar changes caused by ACR thanks to an oligodendrogenic effect of omega 3. (Folia Morphol 2019; 78, 3: 564-574)

Key words: acrylamide, cerebellum, omega 3, green tea, rats, oligodendrocytes

Address for correspondence: Dr. R.A. Imam, Anatomy and Embryology Department, Faculty of Medicine, Cairo University, Cairo, Egypt, tel: 01006114696, e-mail: redaabdelnasser@yahoo.com 


\section{INTRODUCTION}

Acrylamide (ACR) is an industrial neurotoxic chemical that has been found in carbohydrate-rich foods cooked at high temperatures such as potato chips [15]. Therefore, humans are widely exposed to ACR and its neurotoxicity in humans is a significant public health issue attracting wide attention [19]. It was also associated with carcinogenicity and reproductive toxicity [6]. The neurotoxicity of ACR has been known to affect nerve terminal and cysteine residues on the functionally important presynaptic proteins, resulting in inhibition of neurotransmitter release and eventual process degeneration $[25,26]$. Cerebellum controls the maintenance of equilibrium (balance), influences posture and muscle tone as well as coordinating movement [8]. Cerebellar ataxia may be acquired from exposure to many toxic materials [39]. Myelin is an electrical insulator that increases conduction velocity of nerve fibres and is the physical basis for rapid saltatory conduction (in which impulses jump from one node of Ranvier to another) [29]. Inflammatory damage to the oligodendroglia and white matter is involved in the pathogenesis of demyelinating diseases such as multiple sclerosis [5]. Oligodendrocyte is the cell responsible for producing central nervous system (CNS) myelin by the concentric layers of its plasma membrane [34]. It is located in both the grey and the white matter of the CNS: interfascicular oligodendrocytes (white matter) and satellite oligodendrocytes (grey matter only) [14].

The observation that Eskimos had a very low incidence of atherosclerosis and its complications, had led to the first indication of a protective effect of fish oil on atherosclerosis. Today, fish oil is one of the most popular nutraceuticals available in health food stores [27]. Surprisingly, fish oil preparations are also anti-arrhythmic, particularly in patients who have already suffered a myocardial infarction (MI) [9]. In addition to the treatment of hypertriglyceridaemia, a preparation of omega 3-acid ethyl esters is licensed in the United Kingdom for the prevention of recurrent events after MI [32]. Recently, the combined treatment of fish oil dietary supplement and omega 3 polyunsaturated fatty acids injections had been proved to promote post-traumatic brain injury (TBI) restorative processes in the brain, including generation of immature neurons, microvessels, and oligodendrocytes, each of which was significantly correlated with the improved cognitive recovery [30].

Green tea extract had been partially efficacious in preventing neurodegeneration in the brain of lead-treated rats, which resulted from its inhibitory effect on free radical chain reactions generated during oxidative stress caused by lead and from an increase in antioxidant enzyme capacity [23]. Over the last two decades polyphenols, one of green tea constituents, have drawn attention as promising natural dietary molecules for the prevention of ageing and neurodegenerative diseases [22]. Recently, dietary polyphenols, endowed of antioxidant and anti-inflammatory properties, had been reported to extend brain health span as they were actively investigated as potential adjuvants to support proliferation and survival of neural progenitors, and counteract age-dependent neurogenic decline [36].

The present study deals with the influence of green tea extract and omega 3 fatty acids in ACR-induced adverse cerebellar changes in rats.

\section{MATERIALS AND METHODS}

\section{Animals}

The experiment was ethically approved by the Institutional Animal Care and Use Committee of Cairo University (CU-IACUC) under the number of CU/ $/ I I I / F / 48 / 18$. The present study was carried out on 24 male albino rats weighing 150-200 g. The animals were housed in cages, under standard laboratory and environmental conditions with free access to food and water at a temperature of $\left(20 \pm 2^{\circ} \mathrm{C}\right)$ with a natural 12-h light/dark cycle and free access to standard pellet chow and drinking water ad libitum. Animals were obtained from Animal House, Faculty of Medicine, Cairo University. The rats were set in the laboratory for a period of 2 weeks for acclimatisation before carrying out the experiment.

\section{Chemicals}

Acrylamide. The dose of ACR used was $45 \mathrm{mg} / \mathrm{kg} /$ /day [31]. Acrylamide (99\% pure) was purchased from Sigma Chemical Company (St. Louis, Missouri, USA). It was dissolved in distilled water and administered every day by oral gavage.

Omega 3 plus. Omega 3 fatty acids (Sedico, Egypt) was given in a dosage of $(200 \mathrm{mg} / \mathrm{kg} / \mathrm{day}$; DHA $100 \mathrm{mg} / \mathrm{kg} /$ day + EPA $100 \mathrm{mg} / \mathrm{kg} /$ day) which was administered every day by oral gavage [38].

Green tea. Green tea. Royal Regime Tea, packed in Egypt by Royal Herbs, was dissolved in the drinking water at a concentration of $5 \mathrm{~g} / \mathrm{L}$. Tea was prepared freshly 3 times per week and stored at $48^{\circ} \mathrm{C}$ until use. The content of drinking vessels was renewed every day [23]. 


\section{Experimental design}

The rats were randomly divided into four equal groups (6 rats each) as follows:

- Group I (control group): the rats received no medications;

- Group II: the rats received ACR;

- Group III: the rats received ACR concomitant with omega 3;

- Group IV: the rats received ACR concomitant with green tea.

All animals were sacrificed after 8 weeks (the end of the experiment) by decapitation using guillotines to avoid brain injury [10]. Just before sacrification, the body weight of all animals was measured. After euthanasia of animals, the cerebellum of all animals was immediately removed and underwent the following:

\section{Biochemical analysis}

The cerebellum of all animals was immediately washed in ice-cold glass slides, homogenised separately in 10 volumes $(\mathrm{w} / \mathrm{v})$ of $0.1 \mathrm{M}$ phosphate buffer, $\mathrm{pH} 7.4$ using a polytron homogenizer for $1 \mathrm{~min}$. The homogenates were centrifuged at $4000 \mathrm{rpm}$ for $20 \mathrm{~min}$ and refrigerated at $4^{\circ} \mathrm{C}$. The supernatant was used for estimation of the quantitative activities of both malondialdehyde (MDA) and the total glutathione (GSH).

\section{Histological and immunohistochemical study}

Specimens from the cerebellum were fixed in 10\% neutral-buffered formalin. Sections of $5 \mu \mathrm{m}$ thickness were prepared from each specimen. The sections were stained with haematoxylin and eosin (H\&E) as well as the silver stain for histological assessment. For silver staining [2], sections were deparaffinised, treated with $1 \%$ potassium permanganate, bleached in $1 \%$ oxalic acid, treated with $2.5 \%$ iron alum, placed in a Coplin jar of silver solution, reduced in $10 \%$ aqueous formalin, toned in $0.2 \%$ gold chloride solution, treated with $5 \%$ sodium thiosulfate, counterstained with eosin, dehydrated through ascending grades of alcohol then cleared in xylene.

Deparaffinised sections were prepared for immunohistochemical study $[24,35]$. They were mounted on positively charged slides for staining with platelet-derived growth factor alpha (PDGF $\alpha$; as a marker for oligodendrocytes), glial fibrillary acidic protein (GFAP; to assess for astrocytic activity) and BCL2 (as a measure for antiapoptosis). The deparaffinised sections underwent rehydration, heat-induced epitope retrieval (HIER). The slides were placed on
Dako autostainer instrument, EnVision Flex peroxidase blocked. Immune reactions were evaluated with various primary antibodies PDGF $\alpha$ (Genetex, USA), BCL2 (Dako, Denmark), GFAP (DAKO, Denmark). Secondary antibodies used were Dako EnVision Flex/HRP. Visualisation had been done with EnVision FLEX DAB + chromogen. The prepared sections were examined and photographed using a Canon digital camera (Canon, Japan) attached to the IBM computer system.

\section{Histomorphometric study}

Image analysis was performed using the software Leica Quin 500, Germany. It was used to measure the areas per cent of positive PDGF, GFAP and BCL2 immuno-staining reaction in a standard measuring frame using a magnification $\times 400$ by light microscopy transferred to the monitor's screen. These areas were masked by a green colour using the computer system. Area per cent values for each group were obtained from 6 different fields from different slides. Values were presented as a mean and standard deviation and statistically analysed. For optical density of silver staining, the video signals from the video camera were transmitted to the image capture board which digitally converted light intensity to 256 possible grey levels. The image was broken up into discrete picture elements or pixels, with each pixel assigned a specific grey colour. These grey levels ranging from pure black to pure white were automatically converted to optical density values and stored. The number of Purkinje cells for each group was counted by the computer system using a magnification $\times 400$ from 6 different fields from different slides. Values were presented as a mean and standard deviation and statistically analysed.

\section{Statistical analysis}

Statistical analysis was performed using statistical package for the social sciences (SPSS) version 21.0 (IBM Corporation, Somers, NY, USA) statistical software. The data were expressed as means \pm standard deviation (SD). Statistical evaluation was done using one-way analysis of variance (ANOVA) followed by post hoc Tukey test. Significance was considered when $p$ value was less than 0.05 .

\section{RESULTS}

\section{Clinical observations and rats body weight}

The acrylamide group of rats became progressively less active and showed general weakness with a decrease in their appetite from the first time of treatment 
Table 1. Comparison of the body weight, number of Purkinje cells and oxidative/antioxidative markers between the different groups

\begin{tabular}{lcccc}
\hline Group & Body weight & Number of Purkinje cells & GSH [mmol/mg] & MDA [nmol/mg] \\
\hline Control & $217.3 \pm 23.9$ & $10.8 \pm 1.8$ & $55.0 \pm 6.3$ & $2.4 \pm 0.6$ \\
ACR & $157.0 \pm 13.0^{*}$ & $4.74 \pm 0.7^{*}$ & $30.8 \pm 4.2^{* *}$ & $12.9 \pm 1.9^{*}$ \\
ACR + omega 3 & $204.4 \pm 20.0^{* *}$ & $11.26 \pm 1.8^{* *}$ & $41.3 \pm 4.2^{* * *}$ & $5.4 \pm 1.2^{* * * *}$ \\
ACR + green tea & $169.9 \pm 24.7^{* * * *, *}$ & $9.8 \pm 2.6^{* *}$ & $46.6 \pm 5.6^{* * *}$ & $4.0 \pm 0.61 .11^{* * * *}$ \\
\hline
\end{tabular}

Data are shown as mean \pm standard deviation. ACR — acrylamide; GSH — glutathione; MDA — malondialdehyde; * statistically significant as compared to control group; ** statistically

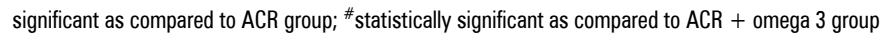

with the drug. Although no mortality was recorded, this group showed a decline in their body sizes. The ACR + omega 3 and ACR + green tea groups were more active with slight decrease in their appetite with no mortality recorded. The body weight of the ACR and $A C R+$ green tea groups was significantly decreased as compared to the control group. It was significantly increased in the ACR + omega 3 group as compared to the ACR and ACR + green tea group (Table 1).

\section{Biochemical results}

The mean GSH of the ACR group was significantly decreased as compared to the control group. It was significantly increased in the ACR + omega 3 and ACR + green tea group as compared to the ACR group (Table 1). The mean MDA of the ACR group was significantly increased as compared to the control group. It was significantly decreased in the ACR + omega 3 and $A C R+$ green tea group as compared to the ACR group (Table 1).

\section{Histological results}

On H\&E (Fig. 1) staining, the control group showed regularly arranged Purkinkje cells and granular cells. The ACR group exhibited regular Purkinje cells alternating with swollen degenerated ones with pale chromatin materials in their nuclei. Some Purkinje cells were seen displaced in the granular layer. The ACR group also exhibited degenerated Purkinje cells surrounded with empty spaces. The granular cells were markedly diminished in number with clumps of pyknotic cells with intercellular eosinophilic areas (necrosis). The ACR + omega 3 group showed largely preserved Purkinje cells with apparently intact all layers of the cortex. Many specimens of this group showed multilayers of Purkinje cells surrounded by many vacuolated cells (clear cells) mostly oligodendrocytes. The ACR + green tea group showed largely preserved Purkinje cells with all layers of the cortex apparently intact.
On silver staining (Fig. 2) of the cerebellar cortex, the ACR group revealed marked loss of Purkinje cells with nuclear degeneration in the remaining ones. The $\mathrm{ACR}+$ omega 3 group revealed a multilayer deposition of regular Purkinje cells with oligodendrocytes inbetween. Granular cells appeared regular despite their decreased staining. The ACR + green tea group revealed largely preserved Purkinje and granular cell layers. On silver staining of the cerebellar medulla, the control group exhibited normal silver staining of the axons while the ACR group exhibited markedly decreased axonal staining. The ACR + omega 3 group exhibited mildly decreased axonal staining while the ACR + green tea group exhibited normal axonal staining.

\section{Immunohistochemical results}

On PDGF immunostaining (Fig. 3), the control group showed many oligodendrocytes with a positive PDGF reaction while the ACR group showed few oligodendrocytes with decreased reaction. The ACR + omega 3 group exhibited an increased number of oligodendrocytes with a positive PDGF reaction, especially at the junction between molecular and granular cell layers, while the ACR + green tea group exhibited a considerable amount of oligodendrocytes with a positive reaction.

On GFAP immunostaining (Fig. 4), the control group showed a considerable number of astrocytes with a positive GFAP reaction while the ACR group showed a huge number of astrocytes with a positive reaction. The ACR + omega 3 group exhibited a considerable number of astrocytes with a positive GFAP reaction while the ACR + green tea group exhibited mild increase in the number of astrocytes with positive GFAP reaction.

On BCL2 immunostaining (Fig. 5), the control group showed mild positive $B C L 2$ reaction while the $A C R$ group showed a scanty $B C L 2$ reaction. The $A C R$ + omega 3 group showed mild increase in BCL2 reaction while the $A C R+$ green tea group exhibited mild positive $B C L 2$ reaction. 

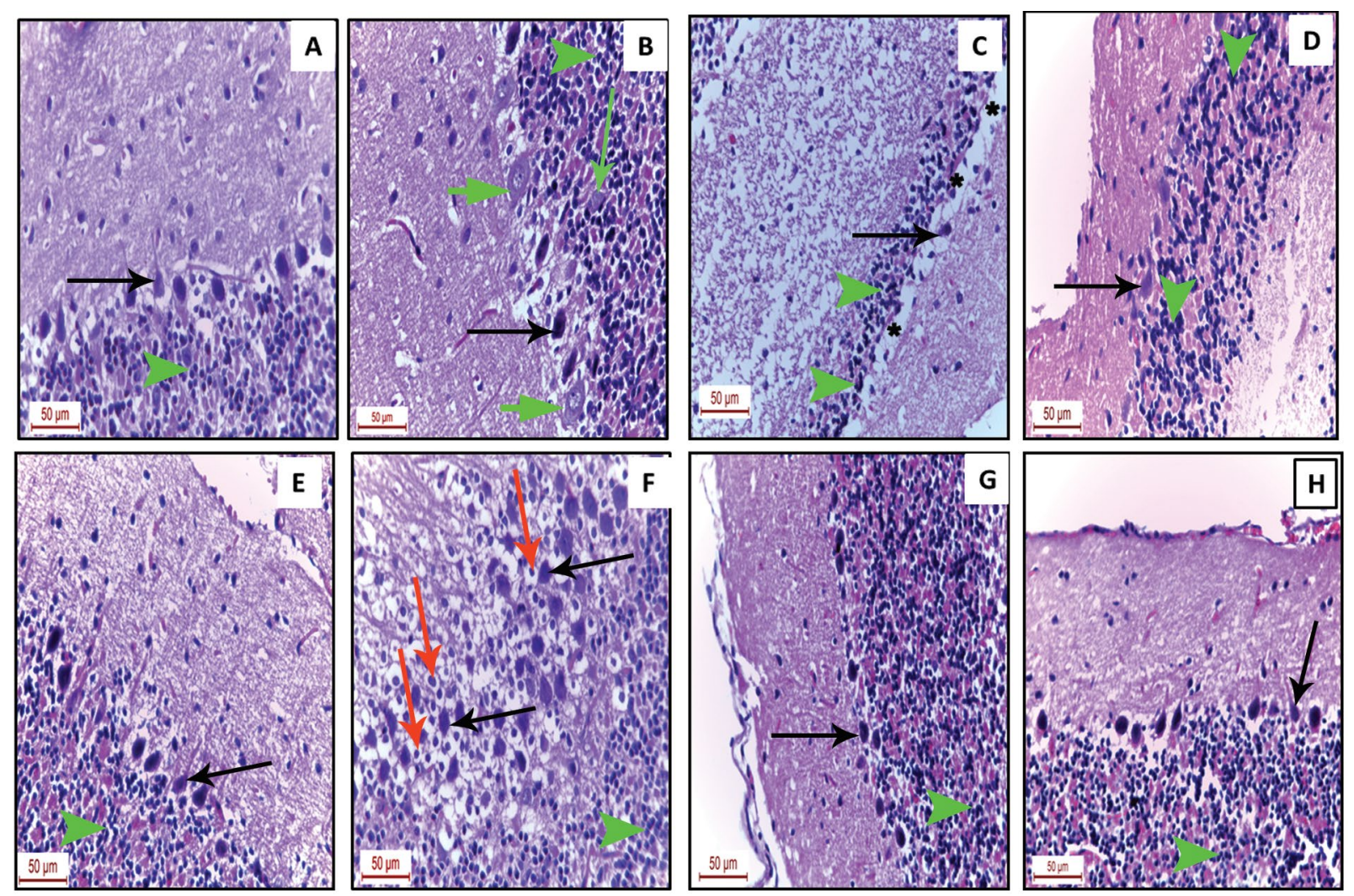

Figure 1. A. Control group showing regularly arranged Purkinkje cells (black arrow) and granular cells (arrow head); B. Acrylamide (ACR) group showing regular Purkinje cells (black arrow) alternating with swollen degenerated ones with pale chromatin materials in their nuclei (incomplete arrow). Purkinje cells (green arrow) are seen displaced in the granular layer and surrounded with dark granular cells (arrow head); C. ACR group showing degenerated Purkinje cells surrounded with empty spaces $\left({ }^{*}\right)$. Purkinje and granular cells are markedly diminished in number; D. ACR group showing degenerated Purkinkje cells and clumps of pyknotic granular cells (arrow heads) with intercellular eosinophilic areas (necrosis); E. Omega 3-treated group showing largely preserved all layers of the cortex; F. Omega 3-treated group showing multilayers of Purkinje cells surrounded by many cells with clear cytoplasm mostly oligodendrocytes (red arrows). The granular cells appear preserved; G, H. Green tea-treated group showing largely preserved all layers of the cortex; haematoxylin and eosin, $\times 400$.

\section{Histomorphometric results}

The mean number of Purkinje cell in the ACR group was significantly decreased as compared to the control group. It was significantly increased in the ACR + omega 3 and $A C R+$ green tea group as compared to the ACR group (Table 1).

The optical density of silver stain was significantly decreased in the ACR group as compared to the control group. It was significantly increased in the ACR + omega 3 and $A C R+$ green tea group as compared to the ACR group (Fig. 6).

The area per cent of $B C L 2$ positive reaction was significantly decreased in ACR group as compared to control group. There was a significant difference between the ACR + omega 3 group as compared to Group IV. There was a significant difference between the ACR + omega 3 group as compared to the ACR group and no significance between the ACR + green tea group as compared to the ACR group (Fig. 6).
The area per cent of PDGF positive reaction was significantly decreased in the ACR group as compared to the control group. It was significantly increased in the ACR + omega 3 as compared to all groups. The $A C R+$ green tea group showed significant increase as compared to the ACR group (Fig. 6).

The area per cent of GFAP positive reaction was significantly increased in the ACR group as compared to the control group. It was significantly decreased in the ACR + omega 3 and group IV as compared to the ACR group (Fig. 6).

\section{DISCUSSION}

Human beings and animals are exposed every day simultaneously and concurrently to environmental contaminants. Acrylamide is known to exert its toxicity through oxidative stress by generating reactive oxygen species [21]. Body weight is an important marker in toxicology experiments. In our study, ACR admin- 

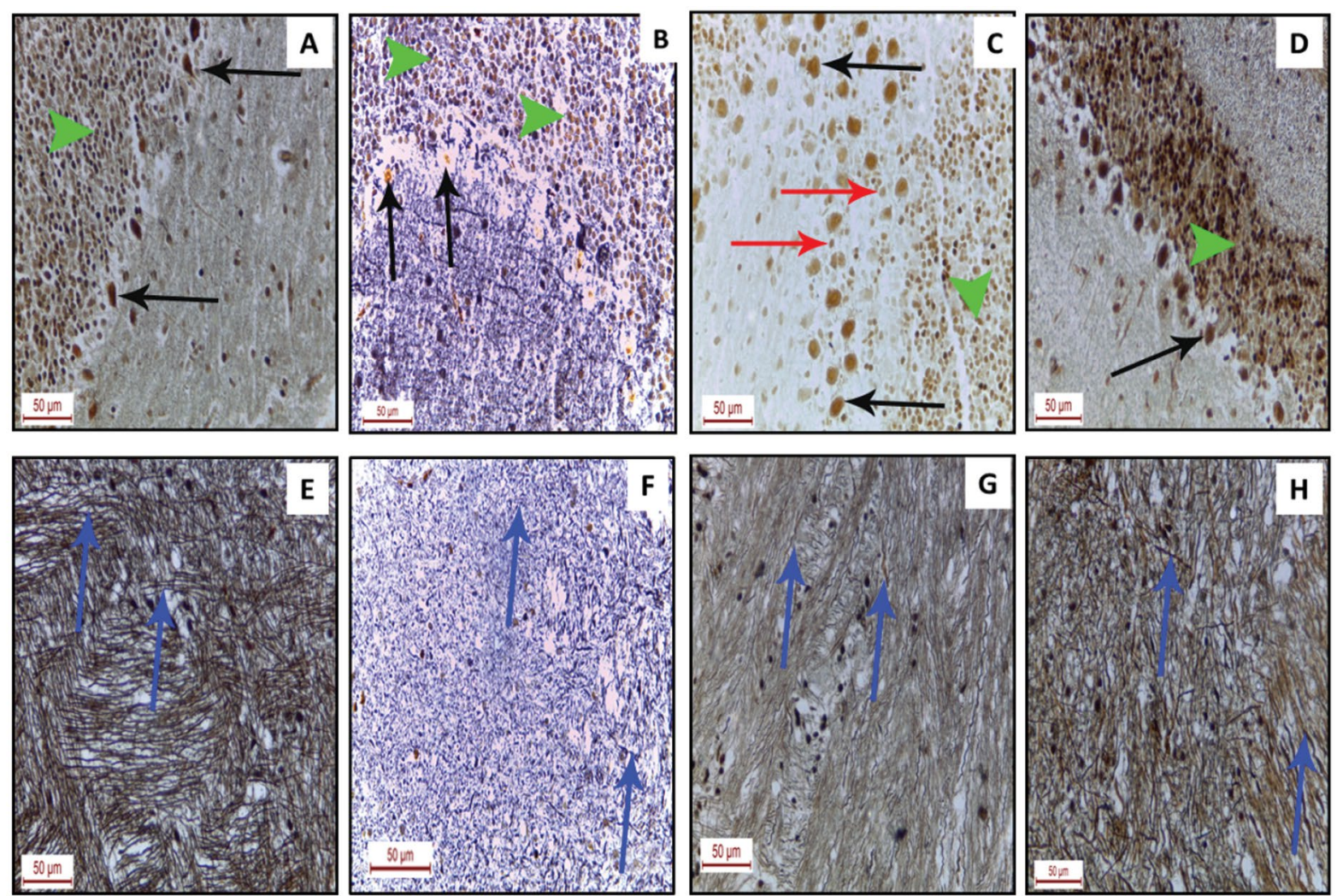

Figure 2. A. Control group showing regularly arranged granular cells (arrow head) and flask shaped Purkinkje cells (black arrows); B. Acrylamide (ACR) group showing loss of Purkinje cells with nuclear degeneration in the remaining ones (black arrow). The granular cells (arrow heads) appear with decreased staining and wide intercellular spaces; C. Omega 3-treated group showing multilayers of regular Purkinje cells with oligodendrocytes (red arrows) inbetween. Granular cells appear regular despite their decreased staining; D. Grean tea-treated group showing largely preseved Purkinje cells and granular layer; E. Control group cerebellar medulla showing normal silver staining of axons (blue arrows); F. ACR group cerebellar medulla showing markedly decreased silver staining of axons; G. Omega 3-treated group cerebellar medulla showing mildly decreased silver staining of axons in the cerebellum; H. Green tea-treated group cerebellar medulla showing normal silver staining of axons; silver $\times 400$.
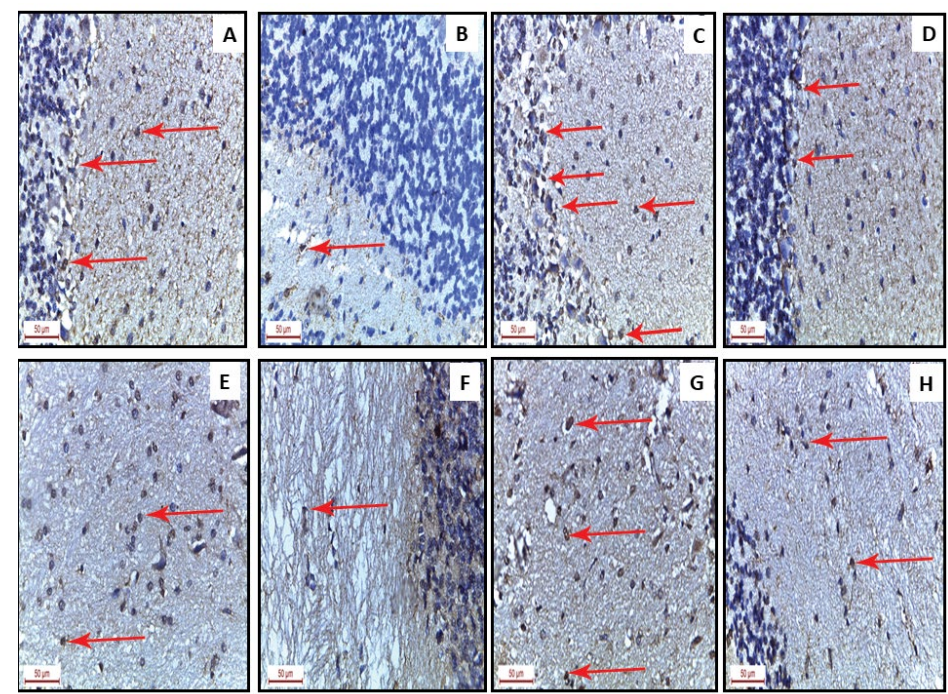

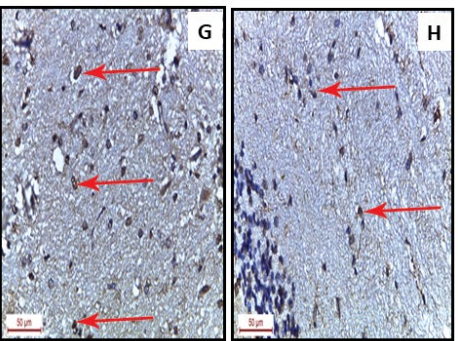

Figure 3. A, E. Control group (cerebellar cortex and medulla respectively) showing many oligodendrocytes (arrows) with positive platelet-derived growth factor (PDGF) reaction; B, F. Acrylamide (ACR) group cerebellar (cerebellar cortex and medulla respectively) showing few oligodendrocytes (arrows) with positive reaction; C, G. Omega 3-treated group (cerebellar cortex and medulla respectively) showing many oligodendrocytes with positive reaction especially at the junction between molecular and granular cell layers; D, H. Green tea-treated group (cerebellar cortex and medulla respectively) showing a considerable amount of oligodendrocytes with positive reaction; PDGF; $\times 400$. 

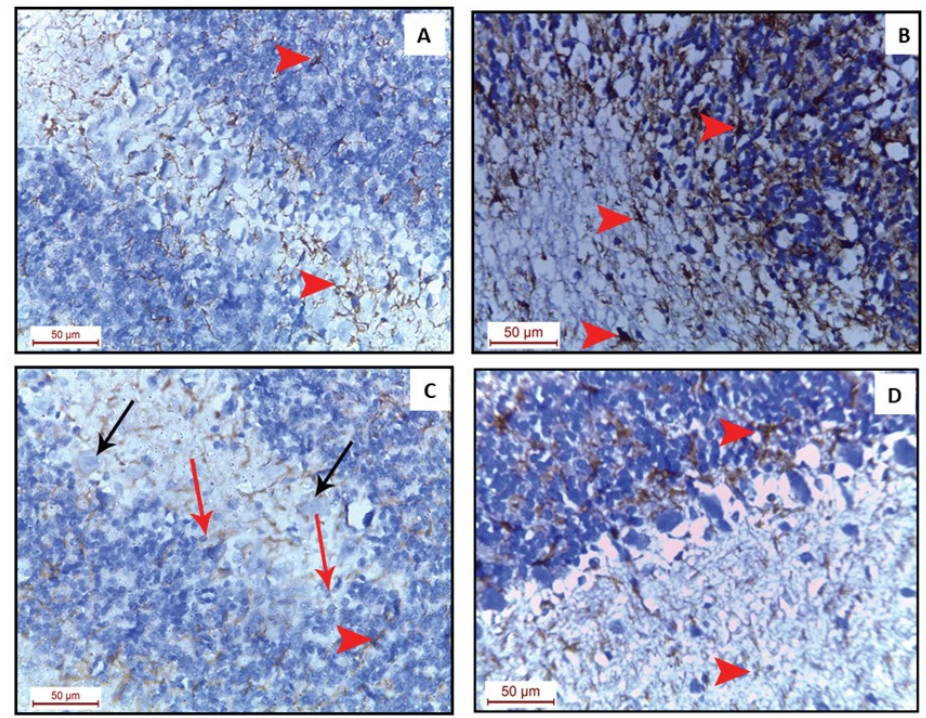

Figure 4. A. Control group showing considerable number of astrocytes (arrow heads) with positive glial fibrillary acidic protein (GFAP) reaction. B. Acrylamide (ACR) group showing huge number of astrocytes with positive reaction; C. ACR + omega 3-treated group showing considerable number of astrocytes with GFAP reaction. Note the cells (red arrow) with negative GFAP reaction at the junction of molecular layer and Purkinje cell (black arrow) layer are mostly oligodendrocytes; D. ACR + green tea-treated group showing mild increase in the number of astrocytes with positive GFAP reaction; GFAP, $\times 400$.
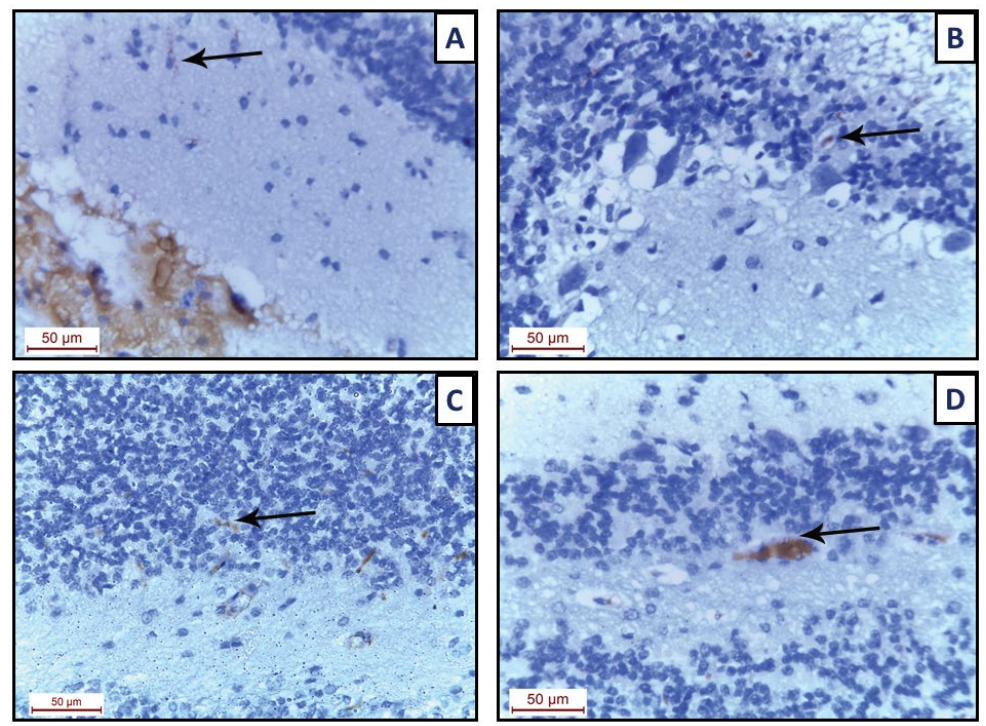

Figure 5. A. Control group showing mild positive $B C L 2$ reaction (arrow); B. acrylamide (ACR) group showing scanty BCL2 reaction; C. Omega 3-treated group showing mild increase in BCL2 reaction; D. Green tea-treated group showing mild positive BCL2 reaction; BCL2, $\times 400$. istered to adult rats reduced the body weight. This might be due to a decrease in food and water intake by adult rats due to decreased appetite [1]. Moreover, ACR toxicity could affect absorption or metabolism of the food, resulting in a decrease in body weight [37].

The significant increase in MDA levels in the ACR group might be due to high content of lipids in the cerebellum which makes lipid peroxidation the main landmark of brain oxidative stress [42]. The decline in GSH levels in the ACR group might reflect its consumption through oxidative stress [7, 12, 41]. Supporting our results, it was reported that ACR had enhanced oxidative stress through increased formation of reactive oxygen species and lipid peroxidation while decreased superoxide dismutase activity and glutathione levels [19].
Histologically, the ACR group exhibited variable degrees of cellular degeneration, especially Purkinje cells, confirmed by the significant decrease in their number as compared to the control group. In agreement to these results, it was observed undifferentiated Purkinje cells with frequent pyknotic ones, a loss of their number and also marked oedema in the upper part of the internal granular layer in the cerebellum of drug-treated rats $[15,18]$. The observed migration of Purkinje cells into the granular layer in this study might be due to the fact that ACR delays the cell proliferation in the granular layer as well as migration and differentiation [1]. These changes might be explained by the possible action of ACR to induce alterations in the cytoskeleton, membrane necrosis, free radicals, oxidative stress and mitochondrial dysfunction [41]. 

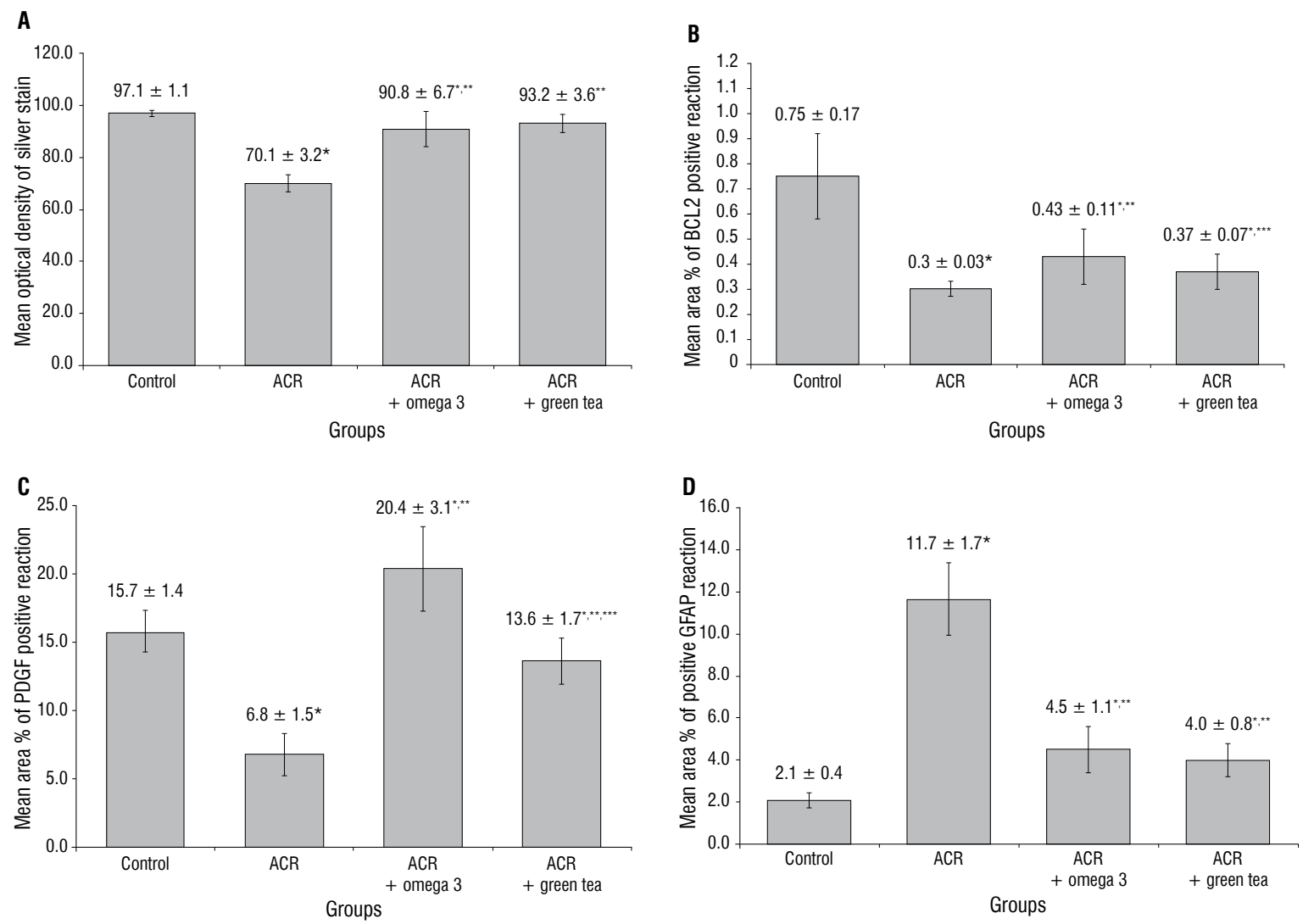

Figure 6. Bar charts of the mean; A. Optical density of silver stain; B. Area per cent of positive BCL2 reaction; C. Area per cent of platelet-derived growth factor (PDGF) positive reaction; D. Area per cent of glial fibrillary acidic protein (GFAP) positive reaction; * statistically significant as compared to Group I; ${ }^{* *}$ statistically significant as compared to Group II; ${ }^{* *}$ statistically significant as compared to Group III.

In this study, some specimens in Group II showed clumping of the granular layer with areas of necrosis inbetween the cells. Partially supporting these findings, it was revealed that chronic ACR administration had led to thinning of the external granular layer, which in turn delays the proliferation of the cells of this layer [1].

On GFAP immunostaining, Group II showed stong positive GFAP reaction in our study. GFAP is an important skeleton protein of astrocytes [37]. Up-regulation of GFAP is associated with proliferation and activation of astrocytes. Almost all types of brain injury may stimulate the upregulation of GFAP in reactive astrocytes, so GFAP could be used as a marker of CNS injuries. ACR had been reported to upregulate the expression of GFAP in the cerebellum in response to the Purkinje cells damage [40]. On BCL2 immunostaining of this work, although mild reaction was observed in all groups, the area per cent of positive reaction in ACR group was significantly decreased as compared to the control group. Supporting this result, it was reported that BCL2 mRNA expression had significantly decreased in PC12 cells treated with ACR implicating increased apoptosis [19].

The omega 3-treated group showed significantly improved results in comparison with those in the ACR group. Histochemically, there was significant decrease in cerebellar MDA level of omega 3-treated rats as compared to the ACR-treated ones. Also, there was significant increase in cerebellar glutathione level of omega 3-treated rats as compared to the ACR group. Concordantly, omega 3 fatty acid supplementation elevated superoxide dismutase and catalase activity in various organs such as the kidney, liver, and intestine [4]. In addition, omega 3 had significantly increase GSH and neuroprotectin D1 (NPD1) in the brain of posttraumatic brain injury in rats [37].

Oligodendrocytes precursor cells are present in the adult CNS and have NG2 and PDGF $\alpha$ receptors [13]. Omega 3 had promoted oligodendrogenesis in the cerebellar cortex in Group III of this work expecially at the junctional area between molecular and granular cell layers, confirmed by their positive PDGF $\alpha$ immunostain and negative GFAP immunostain to exclude 
ACR-induced Bergmann astrocytosis. Confirming these findings, the area per cent of PDGF positive reaction in this work was significantly increased in Group III as compared to all groups. Supporting our results, the combined treatment of fish oil dietary supplement and omega 3 polyunsaturated fatty acids injections had been proved to promote post-TBI restorative processes in the brain, including generation of immature neurons, microvessels, and oligodendrocytes, each of which was significantly correlated with the improved cognitive recovery [30]. Oligodendrocytes were abundant in H\&E sections of omega 3 plus-treated rats in this work. Oligodendrocytes are identified histologically by having condensed, rounded nuclei and unstained cytoplasm due to very abundant Golgi complexes, which stain poorly [28]. The observed oligodendrocytes in this work were in the grey matter of cerebellum in its molecular layer (i.e. satellite oligodendrocytes). It was suggested that the function of this type of oligodendrocytes is to monitor the extracellular fluid around neuronal cell bodies, act in a reserve capacity, and, if the need arises, they may migrate into the white matter to replenish interfascicular oligodendrocytes [14].

Omega 3 polyunsaturated fatty acids ( $n-3$ PUFAs) exist abundantly in the brain and play a crucial role in essential neuronal functions, such as axonal guidance, synapse and dendrite formation, neurotransmission, etc. $[17,20]$. Other mechanisms by which omega 3 polyunsaturated fatty acids exert potent protective effects following experimental TBI had been reported, for example, amelioration of oxidative stress [33], mitigation of endoplasmic reticulum stress [3] and modulation of microglial activation [16].

The present work showed improvement in the histopathological findings with the co-administration of green tea extract with ACR. Interestingly, the protective effects of green tea extract against neurotoxicity caused by ACR exposure, both in vivo and in vitro, was attributed to antioxidant effects of this extract [11]. Both epigallocatechin gallate (EGCG) and epicatechin gallate (ECG), which are green tea catechins, showed inhibitory effects on ACR neurotoxicity. They increased GSH level and decreased lipid peroxidation in rat cerebral cortex [12]. A study on the PC12 cell line of pheochromocytoma revealed that EGCG attenuates ACR-induced neurotoxicity in PC12 cells by maintaining mitochondrial function and regulating the expression of $\mathrm{BAX}$ and $\mathrm{BCL} 2 \mathrm{mRNA}$ and redox state [19].
Optical density of silver staining in this work was significantly lower in the ACR-treated group as compared to the control. This might indicate the demyelinating effect of ACR. Having the major role in myelinating central axons, each oligodendrocyte can myelinate individual intermodal segments of an average of 30 separate axons (as high as 60 axons); adjacent internodal segments are myelinated by different oligodendrocytes [13]. This pattern of central myelination leaves periodic nodes of Ranvier bare, separating between adjacent segments, with sodium channels, at which action potentials are reinitiated as they travel down the myelinated axon and its branches (called saltatory conduction) $[8,13]$. Both omega 3 and green tea significantly increased the optical density of silver staining of the cerebellar axons as compared to ACR-treated rats in the current work; this might suggest their myelinating role and their potential oligodendrocytes recruitment. Oligodendrocytes can be attacked by antibodies directed at specific oligodendrocyte proteins in multiple sclerosis, leading to oligodendrocyte death and axonal dysfunction [13]. Also, they could be attacked by a polyoma virus (JC virus) causing demyelinisation of axons especially in the occipital and parietal lobes of the brain leading to progressive multifocal leukoencephalopathy [14].

Clinically implicated from the current work, omega 3 might have an oligodendrogenic role and might be used in CNS injury to help myelin sheath regeneration. However, the exact mechanism by which omega 3 induces oligodendrogenisis is not clear and still needs further investigations. Also, this oligodendrogenic role after already established CNS injury should be thoroughly investigated.

\section{CONCLUSIONS}

In conclusion, ACR causes adverse cerebellar changes. Concomitant administration of omega 3 or green tea with ACR might mitigate these adverse changes thanks to an oligodendrogenic effect of omega 3.

\section{REFERENCES}

1. Allam A, El-Ghareeb AA, Abdul-Hamid M, et al. Prenatal and perinatal acrylamide disrupts the development of cerebellum in rat: Biochemical and morphological studies. Toxicol Ind Health. 2011; 27(4): 291-306, doi: $10.1177 / 0748233710386412$, indexed in $\mathrm{Pu}$ bmed: 21310778.

2. Bancroft JD, Layton C. Connective and other mesenchymal tissues with their stains. In: Suvarna SK, Bancroft, JD, 
Editors. Bancroft's Theory and Practice of Histological Techniques, Eighth Edition, Elsevier Limited. 2019: 53-175.

3. Begum G, Yan HQ, Li L, et al. Docosahexaenoic acid reduces ER stress and abnormal protein accumulation and improves neuronal function following traumatic brain injury. J Neurosci. 2014; 34(10): 3743-3755, doi: 10.1523/ JNEUROSCI.2872-13.2014, indexed in Pubmed: 24599472.

4. Brahmbhatt $V$, Oliveira $M$, Briand $M$, et al. Protective effects of dietary EPA and DHA on ischemia-reperfusion-induced intestinal stress. J Nutr Biochem. 2013; 24(1): 104-111, doi: 10.1016/j.jnutbio.2012.02.014, indexed in Pubmed: 22819560.

5. Buja LM, Krueger GRF. Nervous System. In:, Krueger GRF editors. 2nd edition. Netter's Illustrated Human Pathology, Saunders, an imprint of Elsevier Inc, Philadelphia, 2014: 441-513.

6. Carere A. Genotoxicity and carcinogenicity of acrylamide: a critical review. Ann Ist Super Sanita. 2006; 42(2): 144-155, indexed in Pubmed: 17033134.

7. Catalá A. The ability of melatonin to counteract lipid peroxidation in biological membranes. Curr Mol Med. 2007; 7(7): 638-649, indexed in Pubmed: 18045142.

8. Crossman AR, Neary D. Cells of the nervous system. In:, editors. Neuroanatomy: An Illustrated Colour Text, 5th edition, Churchil Livingstone an imprint of Elsevier Limited. 2015: 32-35.

9. Dominiczak MH. Lipoprotein Metabolism and Atherogenesis. In: Baynes JW, Hab M, editors. Medical Biochemistry, 5th edition, Elsevier, Saunders. 2019: 489-506.

10. Erasmus MA, Lawlis $P$, Duncan $\mathrm{IJH}$, et al. Using time to insensibility and estimated time of death to evaluate a nonpenetrating captive bolt, cervical dislocation, and blunt trauma for on-farm killing of turkeys. Poult Sci. 2010; 89(7): 1345-1354, doi: 10.3382/ps.2009-00445, indexed in Pubmed: 20548061.

11. Esmaeelpanah E, Rahmatkhah A, Poormahmood N, et al. Protective Effect of Green Tea Aqueous Extract on Acrylamide Induced Neurotoxicity. Jundishapur J Nat Pharm Prod. 2015; 10(2), doi: 10.17795/jjnpp-18406.

12. Esmaeelpanah E, Razavi BM, Vahdati Hasani $F$, et al. Evaluation of epigallocatechin gallate and epicatechin gallate effects on acrylamide-induced neurotoxicity in rats and cytotoxicity in PC 12 cells. Drug Chem Toxicol. 2018; 41(4): 441-448, doi: 10.1080/01480545.2017.1381108, indexed in Pubmed: 29072525.

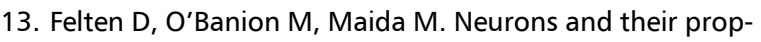
erties. 3rd edition. Netter's Atlas of Neuroscience. 2016: 1-42, doi: 10.1016/b978-0-323-26511-9.00001-1.

14. Gartner LP. Nervous tissue In: Textbook of Histology, 4th edition, Elsevier, Philadelphia. 2017: 211-249.

15. Ghorbel I, Amara IB, Ktari N, et al. Aluminium and Acrylamide Disrupt Cerebellum Redox States, Cholinergic Function and Membrane-Bound ATPase in Adult Rats and Their Offspring. Biol Trace Elem Res. 2016; 174(2): 335-346, doi: 10.1007/s12011-016-0716-1, indexed in Pubmed: 27116954.

16. Harvey LD, Yin Y, Attarwala IY, et al. Administration of DHA Reduces Endoplasmic Reticulum Stress-Associated Inflammation and Alters Microglial or Macrophage Activation in Traumatic Brain Injury. ASN Neuro. 2015; 7(6), doi: 10.1177/1759091415618969, indexed in Pubmed: 26685193.
17. Hasadsri L, Wang BH, Lee JV, et al. Omega-3 fatty acids as a putative treatment for traumatic brain injury. J Neurotrauma. 2013; 30(11): 897-906, doi: 10.1089/neu. 2012.2672, indexed in Pubmed: 23363551.

18. Hasebe M, Matsumoto I, Imagawa T, et al. Effects of an anti-thyroid drug, methimazole, administration to rat dams on the cerebellar cortex development in their pups. Int J Dev Neurosci. 2008; 26(5): 409-414, doi: 10.1016/j.ijdevneu.2008.03.007, indexed in Pubmed: 18456449.

19. He Y, Tan D, Mi Y, et al. Effect of epigallocatechin-3-gallate on acrylamide-induced oxidative stress and apoptosis in PC12 cells. Hum Exp Toxicol. 2017; 36(10): 1087-1099, doi: 10.1177/0960327116681648, indexed in Pubmed: 27920337.

20. Hering $H$, Lin CC, Sheng M. Lipid Rafts in the Maintenance of Synapses, Dendritic Spines, and Surface AMPA Receptor Stability. J Neurosci. 2003; 23(8): 3262-3271, doi: 10.1523/jneurosci.23-08-03262.2003.

21. Lu H, Yuan G, Yin Z, et al. Effects of subchronic exposure to lead acetate and cadmium chloride on rat's bone: $\mathrm{Ca}$ and $\mathrm{Pi}$ contents, bone density, and histopathological evaluation. Int J Clin Exp Pathol. 2014; 7(2): 640-647, indexed in Pubmed: 24551284.

22. Hügel HM. Brain food for alzheimer-free ageing: focus on herbal medicines. Adv Exp Med Biol. 2015; 863: 95-116, doi: 10.1007/978-3-319-18365-7_5, indexed in Pubmed: 26092628.

23. Khalaf AA, Moselhy WA, Abdel-Hamed MI. The protective effect of green tea extract on lead induced oxidative and DNA damage on rat brain. Neurotoxicology. 2012; 33(3): 280-289, doi: 10.1016/j.neuro.2012.02.003, indexed in Pubmed: 22342836.

24. Khorramirouz R, Go JL, Noble C, et al. A novel surgical technique for a rat subcutaneous implantation of a tissue engineered scaffold. Acta Histochem. 2018; 120(3): 282-291, doi: 10.1016/j.acthis.2018.02.010, indexed in Pubmed: 29519681.

25. LoPachin RM. The changing view of acrylamide neurotoxicity. Neurotoxicology. 2004; 25(4): 617-630, doi: 10.1016/j. neuro.2004.01.004, indexed in Pubmed: 15183015.

26. LoPachin RM, Barber DS, He D, et al. Acrylamide inhibits dopamine uptake in rat striatal synaptic vesicles. Toxicol Sci. 2006; 89(1): 224-234, doi: 10.1093/toxsci/kfj005, indexed in Pubmed: 16207938.

27. Meisenberg G, Simmons W. The metabolism of membrane lipids. Principles of Medical Biochemistry. 4 th edition. Elsevier, Philadelphia. 2012: 412-423, doi: 10.1016/b9780-323-07155-0.00024-1.

28. Mescher AL. Nerve Tissue \& The Nervous System. In: Junqueira's Basic Histology Text and Atlas, Thirteen's edition, McGraw-Hill Education, USA. 2013: 170.

29. Ovalle WK, Nahirney PC. Nervous Tissue. In: Netter's Essential Histology, 2nd Edition, Elsevier, Philadelphia. 2013: 101-130.

30. Pu H, Jiang X, Wei $Z$, et al. Repetitive and Prolonged Omega-3 Fatty Acid Treatment After Traumatic Brain Injury Enhances Long-Term Tissue Restoration and Cognitive Recovery. Cell Transplant. 2017; 26(4): 555-569, doi: 10.3727/096368916X693842, indexed in Pubmed: 27938482.

31. Rajeh NA, Al-Dhaheri NM. Antioxidant effect of vitamin E and 5-aminosalicylic acid on acrylamide induced kidney injury 
in rats. Saudi Med J. 2017; 38(2): 132-137, doi: 10.15537/ smj.2017.2.16049, indexed in Pubmed: 28133684.

32. Rang HP, Ritter JM, Flower RJ, et al. Rang \& Dale's Pharmacology, 8th edition, Elsevier, Churchil Livingstone, 23; Atherosclerosis and lipoprotein metabolism. 2016: 285-292.

33. Ren $\mathrm{H}$, Yang $\mathrm{Z}$, Luo $\mathrm{C}$, et al. Enriched endogenous omega-3 fatty acids in mice ameliorate parenchymal cell death after traumatic brain injury. Mol Neurobiol. 2017; 54(5): 3317-3326, doi: 10.1007/s12035-016-9931-1, indexed in Pubmed: 27167127.

34. Ross MH, Pawlina W. Histology: a text and atlas - with correlated cell and molecular biology. 7th edition, chapter 12. Wolters Kluwer Health, Philadelphia 2016: 378.

35. Sanderson S, Wild G, Cull AM, et al. Immunohistochemical and immunofluorescent techniques. In: Suvarna SK, Layton C, Bancroft, JD, Editors. Bancroft's Theory and Practice of Histological Techniques, Eighth Edition, Elsevier Limited. 2019: 337-394.

36. Sarubbo F, Moranta D, Pani G. Dietary polyphenols and neurogenesis: Molecular interactions and implication for brain ageing and cognition. Neurosci Biobehav Rev. 2018; 90: 456-470, doi: 10.1016/j.neubiorev.2018.05.011, indexed in Pubmed: 29753753.

37. Shi J, Ma Y, Zheng M, et al. Effect of sub-acute exposure to acrylamide on GABAergic neurons and astrocytes in weaning rat cerebellum. Toxicol Ind Health. 2012; 28(1): 10-20, doi: 10.1177/0748233711401264, indexed in Pubmed: 21444355.

38. Shim JiS, Kim DH, Bae JH, et al. Effects of omega-3 fatty acids on erectile dysfunction in a rat model of atherosclerosis-induced chronic pelvic ischemia. J Korean Med Sci. 2016; 31(4): 585-589, doi: 10.3346/jkms.2016.31.4.585, indexed in Pubmed: 27051243.

39. Subramony $\mathrm{SH}$, Xia G. Disorders of the cerebellum, including the degenerative ataxias. Neurology in Clinical Practice. 2012: 1802-1823, doi: 10.1016/b978-1-4377-0434-1.00097-9.

40. Tanaka H, Katoh A, Oguro K, et al. Disturbance of hippocampal long-term potentiation after transient ischemia in GFAP deficient mice. J Neurosci Res. 2002; 67(1): 11-20, doi: 10.1002/jnr.10004, indexed in Pubmed: 11754076.

41. Yousef MI, El-Demerdash FM. Acrylamide-induced oxidative stress and biochemical perturbations in rats. Toxicology. 2006; 219(1-3): 133-141, doi: 10.1016/j. tox.2005.11.008, indexed in Pubmed: 16343728.

42. Yousef MI. Aluminium-induced changes in hemato-biochemical parameters, lipid peroxidation and enzyme activities of male rabbits: protective role of ascorbic acid. Toxicology. 2004; 199(1): 47-57, doi: 10.1016/j. tox.2004.02.014, indexed in Pubmed: 15125998. 